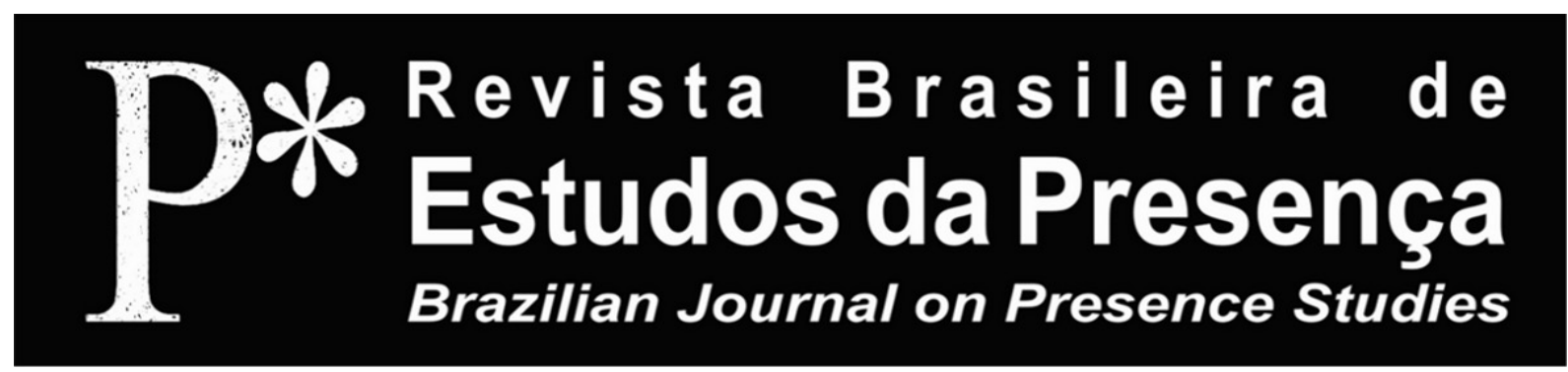

DOI - http://dx.doi.org/10.1590/2237-266036964

ISSN 2237-2660

\title{
Sport, Théâtre et Arts Vivants
}

\author{
Pierre Philippe-Meden \\ Paris 8 Université de Vincennes-Saint-Denis - Paris, France \\ Maison des Sciences de l'Homme Paris Nord - MSH - Paris, France \\ Éléonore Martin \\ Université de La Rochelle - La Rochelle, France \\ Paris 8 Université de Vincennes-Saint-Denis - Paris, France \\ Maison des Sciences de l'Homme Paris Nord - MSH - Paris, France
}

RÉSUMÉ - Sport, Théâtre et Arts Vivants - Rapprocher théâtre et sports paraît paradoxal en Europe, mais pas dans le monde chinois où aucune frontière ne semble exister entre eux. L'histoire révèle l'interpénétration du théâtre et des sports. Or, la catégorisation des sciences fait que des objets résultants de leur fertilisation croisée sont négligés, mais aussi des pans de réalités sociales, économiques et idéologiques, de la vie culturelle, du goût des populations et de leurs industries. Quatre thèmes sont avancés pour aborder cette problématique: l'interpénétration des langues du sport et du théâtre; le théâtre comme ornementation du sport; le sport comme préparation aux métiers du théâtre; la nature entre sports et théâtre.

Mots-clés: Esthétique. Théâtre. Sport. Arts Vivants. Histoire.

ABSTRACT - Sport, Theatre and the Performing Arts - Bringing together theatre and sport may seem paradoxical in Europe, but not in China where there seems to be no boundary between the two. There have also been cross-links between theatre and sport in history. However, scientific categorisation has neglected the products of this cross-fertilization and with it entire social, economic, ideological and cultural realities as well as the interest areas of populations and related industries. We propose four themes to address this issue: cross-links between the language of sport and theatre; theatre as ornament of sport; sport to prepare for theatre professions; synergies between the nature of sport and theatre. Keywords: Aesthetics. Theatre. Sports. Performing Arts. History.

RESUMO - Esporte, Teatro e Artes Cênicas - Aproximar teatro e esportes parece ser paradoxal na Europa, mas não no mundo chinês, onde nenhuma fronteira parece existir entre ambos. A história revela a interpenetraçáo do teatro e dos esportes. Ora, a categorização das ciências faz com que objetos resultantes de sua fertilizaçáo cruzada sejam negligenciados, mas também partes de realidades sociais, econômicas e ideológicas, da vida cultural, do gosto das populações e de suas indústrias. Quatro temas são vislumbrados para abordar essa problemática: a interpenetração das relaçóes entre esporte e teatro; o teatro como ornamentaçáo do esporte; o esporte como preparaçáo para as atividades teatrais; a natureza entre esportes e teatro.

Palavras-chave: Estética. Teatro. Esporte. Artes Cênicas. História. 


\section{Introduction}

Alors que la relation entre l'art et le sport s'entend comme une évidence du XX $\mathrm{XX}^{\mathrm{e}}$ siècle (Daniel, 2009), notamment à travers la peinture des impressionnistes : scènes de plein air et canotiers chez Caillebotte ou scènes de turf chez Degas, ou encore à travers le cinéma d'auteur: Forza Bastia, de Tati (1978), Raging Bull, de Scorsese (1980), Senna, de Kapadia (2010), le rapprochement entre l'univers du théâtre et celui des sports paraît paradoxal. Or, l'histoire des techniques, des sensibilités et des représentations révèle l'intrication constante de ces deux univers depuis la fin du XIX ${ }^{\mathrm{e}}$ siècle et le début $\mathrm{du} \mathrm{XX}^{\mathrm{e}}$ siècle. Mais la catégorisation des sciences et le cloisonnement relatif des disciplines, entre autre des études théâtrales et des études sportives, fait que les objets résultants de la fertilisation croisée entre l'univers du théâtre et celui des sports sont souvent négligés ou ignorés au risque de sous-estimer des pans entiers de réalités sociales, économiques et idéologiques, de la vie culturelle internationale, du goût des populations et de leurs industries. Pourtant, si ce rapprochement paraît étrange dans les zones euro-américaines, il l'est peut-être moins dans le monde chinois.

Le caractère holistique de la pensée cosmologique chinoise, par exemple, rend malaisé l'appréhension de la frontière entre ces deux éléments. La pensée cosmologique établit un rapport entre l'homme et le cosmos, soit une "anthropo-cosmologie" (Cheng, 1997, p. 253) dont l'un des symboles les plus connus et représentatifs est sans aucun doute le Yin/yang:

Le principe de la différence qui crée attraction, ainsi que du devenir et de la multiplicité qu'ils font naître par leurs combinaisons ; mais aussi, par la corrélation étroite qui les unit, ils sont les témoins de l'Unité de fond sous-jacente au monde. Ils illustrent le dynamisme des contraires qu'on ne connaît que par couples, dont l'antagonisme se manifeste par la loi d'alternance qui régit leur fonctionnement (jamais l'un sans l'autre en même temps en un même lieu, sinon l'un virtuellement dans l'autre), qui est réservée, comprimée et intensifiée, en une oscillation mouvante faite de passages constants de l'un à l'autre, de l'un vers l'autre (Robinet, 1991, p. 17).

Ces principes, loin d'être des théories abstraites, se retrouvent donc de manière concrète dans les formes spectaculaires et performatives codifiées classiques, comme le Jingju 京剧 (mieux connu 
sous l'appellation Opéra de Pékin). Le Jingju, originaire de la capitale Beijing, a vu le jour à la fin du XVIII siècle: il se compose de chants, danses, combats, acrobatie, mime, musique, diction (particulière entre le chant et la déclamation). En chinois, l'expression chang唱, nian念, zuo做, da打 c'est-à-dire respectivement chanter, parler (ou déclamer), faire (ici faire est à comprendre en tant que action que l'on pourrait traduire par mime si l'on utilise nos catégories spectaculaires) et se battre renvoie aux éléments indispensables constitutifs du Jingju (Pimpaneau, 1983; Darrobers, 1998). En résumé, chaque action est inséparable d'une autre, à l'image du Yin et du Yang.

Afin d'ouvrir de nouvelles pistes d'études, nous survolerons quatre thématiques significatives des relations entre théâtre et sports: l'interpénétration des langages du théâtre et des sports; le théâtre comme ornementation de l'esprit sportif; le sport comme préparation aux métiers du théâtre; et, enfin, l'identité naturiste entre sports et théâtre.

\section{L'Interpénétration des Langages des Sports et du Théâtre}

De nombreux emprunts de mots, termes et expressions existent entre le milieu du théâtre et l'univers sportif. Les réformateurs et les chercheurs en études théâtrales usent fréquemment de la lexicologie sportive, par exemple: "athlétisme affectif" (Artaud, 1935) et "training de l'acteur" (Müller, 2000), tandis que la terminologie théâtrale se trouve parfois employée en anthropologie du sport pour décrire le spectacle sportif, comme Christian Bromberger pour le match de football (Bromberger, 2009). L'entremêlement des langues théâtrale et sportive est efficient au point de vue des conceptualisations d'expériences, ainsi qu'en inventions poétiques, mais non sans malentendus.

Il arrive souvent que la fréquence d'emploi de certains mots soit inversement proportionnelle à la clarté de leur contenu. On en use comme sans y penser. Cette situation ne s'observe pas seulement dans le langage ordinaire mais aussi à l'intérieur des sciences sociales. On peut y vérifier que certains termes d'usage courant sont, à l'image des mots d'ordre politiques, fort peu définis (Lenclud, 1987, p. 110).

D'après Pierre de Coubertin (1913), le seul talent de JaquesDalcroze est de s'être servi de l'expression: "gymnastique rythmique", dans un temps où les exercices physiques tiennent le haut du pavé 
et où le qualificatif exotique est toujours recherché. Sous le nom de chorégraphie, les jeunes filles s'en seraient détournées. La gymnastique rythmique n'ajoute cependant rien à la danse. Sur un sol déjà ancien, elle revêt les aspects d'un passé dans l'air du temps: mimiques antiques, cortèges, agenouillements, levées des bras et peinture de sentiments simples comme la peur, la joie et l'appétence sexuelle. Dès 1913, au cours du treizième Congrès International d'Éducation Physique, Coubertin aperçoit dans l'avenir la participation des rythmiciennes aux Olympiades pour enguirlander les exploits des athlètes, à condition de chasser d'elles les mouvements trop mous, trop hésitants, trop complexes, trop emprunts de sentimentalisme raffiné pour n'accepter que les plus simples, les plus nets, ceux qui traduisent le mieux des impressions primitives et aisément compréhensives.

Concernant le monde chinois, ce questionnement est délicat. Comme nous l'avons dit précédemment la notion de sport est relativement récente (XIX ${ }^{\mathrm{e}}$ siècle), influencée par le monde occidental. Il en va de même pour le théâtre. A l'aube du XX ${ }^{\mathrm{ème}}$ siècle, un nouveau genre de spectacle apparaît sur le continent chinois: le Huaju 话剧, soit littéralement le théâtre parlé qui est en réalité le théâtre tel qu’il est conçu en Europe. Le Jingju est une forme spectaculaire dite traditionnelle, native de Chine. En 1919, le mouvement du 4 mai (wusi yundong 五四运动), initialement déclenché par les étudiants de Beijing sur la place de Tian'anmen contre les concessions allemandes cédées au japon, reflète une crise intellectuelle profonde. Ainsi

Plus ou moins convertis au genre de vie des Occidentaux, résidant dans les ports ouverts où règne la prospérité artificielle qu'y entretient la présence étrangère, de nombreux intellectuels chinois, et avec eux, la jeunesse des écoles en viendront à penser que le salut de la Chine est dans le rejet total de toutes ses traditions et dans l'imitation systématique de l'Occident. De là, une grande fièvre de savoir et un bouillonnement anarchique des idées et des théories (Gernet, 2003, p. 558).

Le Jingju est alors concurrencé sévèrement par le théâtre parlé en plein essor, notamment par la mise en avant des ouvres d'Ibsen par $\mathrm{Hu}$ Shi, séduit, entre autre, par le réalisme des pièces de l'auteur norvégien (Zhang, 1998, p. 56). L'opposition essentielle retenue entre le Xiqu (dont le Jingju est l'une des sous-catégories) et le théâtre chinois, c'est-à-dire le théâtre parlé Huaju, se joue entre la convention artificielle et le réalisme. Opéra et théâtre parlé apparaissent en 
Chine au XX ${ }^{\text {ème }}$ siècle et sont des formes spectaculaires importées. Le sinologue Vincent Durand-Dastès remarque avec justesse, lorsqu'il s'agit de définir le théâtre chinois:

Il serait donc plus juste de parler de 'théâtre chanté' chinois, même si on ne peut guère éviter le terme d'opéra chinois' tant il est passé dans l'usage. Le terme d'opéra n'a pourtant de sens que s'il existe comme en Occident un 'théâtre parlé' qui s'en distingue. Or un tel genre n'apparut en Chine quau début du $\mathrm{XX}^{\mathrm{e}}$ siècle, et par imitation de modèles occidentaux (Durand-Dastès, 2012, p. 92).

La question d'interpénétration des langages d'un monde à l'autre nécessite de les définir clairement. Le vocabulaire attaché au Jingju est très spécifique et ne s'applique que difficilement à d'autres domaines. Quant au théâtre parlé, une étude spécifique serait à mener.

\section{Le Théâtre comme Ornementation de l'Esprit Sportif}

Les Jeux Olympiques et paralympiques de Londres occupent l'actualité scientifique sportive de l'été 2012, à l'image d'un colloque international qui s'est tenu à Dunkerque les 18 et 19 mai 2012, sur l'idée sportive et l'idée olympique, leurs réalités au XXI ${ }^{e}$ siècle. L'ensemble des programmes de journées d'études, de colloques et de congrès divers organisés autour de l'olympisme manque toutefois de s'intéresser spécialement à l'élément esthétique des cérémonies olympiques, celui de l'ornementation. Ce sujet s'institutionnalise nettement dans un débat tenu en 1906, dans l'enceinte du Théâtre du Peuple, à Bussang, où Coubertin, Maurice Pottecher et Mounet-Sully débattent des meilleurs moyens pour rendre féconde l'association des sports, pensés comme manifestation de la vie physique; et du théâtre comme manifestation de la vie intellectuelle. Entreprise qui s'illustre différemment au cours du $\mathrm{XX}^{\mathrm{e}}$ siècle, chez Rudolf von Laban en prévision des Jeux olympiques de 1936 à Berlin ${ }^{1}$, ou, chez Philippe Decouflé pour les Jeux Olympiques d'Albertville en 1992. L'ornementation de l'esprit sportif par le théâtre suppose encore d'autres rapports. Par ailleurs, dans la plupart des cultures du monde, se trouvent des traces de manifestations spectaculaires qui regroupent un nombre parfois très important de personnes et ceci dans tous les champs de la vie sociale et religieuse, entre autres des sports et des pratiques guerrières, par exemples lors des Fêtes gymniques des Sokols de Tchécoslovaquie ou dans les actuelles chorégraphies de 
masse en Corée du Nord. En France, lorsque Firmin Gémier crée son Edipe, sous le titre Spectacle Olympique (1919), au Cirque d'Hiver, une véritable séance d'athlétisme (courses, sauts, lancers du disque et du javelot, pancrace et jeux acrobatiques) à laquelle participent une centaine de membres de sociétés sportives et quelques recordmen, parmi lesquels Géo André, Paoli et Campana, s'insère pour la première fois dans un drame. Éléments jusqu'alors juxtaposés, sports et théâtre se lient intimement dans les 800 mètres d'Andrey Obey, mis en scène par Jean-Louis Barrault, en 1941, sous le Régime de Vichy, sur le Stade Roland Garros, avec intermède gymnique par la Section Spéciale de Démonstration du Groupement Hébertiste ${ }^{2}$ du Régiment de Sapeur-Pompiers de Paris. La direction artistique de ce spectacle par Barrault fait qu'il sera désormais irrémédiablement associé à la politique collaborationniste de Vichy, mais la performance ne correspond pas aux recherches de la politique sportive de cette période. La performance mise en piste par Barrault est celle des dieux du stade devenus hémiplégiques, se contorsionnant et simulant les pires efforts pour avancer de 10 centimètres (Bonnal, 2000, p. 174-177).

La spectacularisation de la performativité sportive entraîne la mise en performance de la machine actoriale. Ces deux processus conjoints sous-tendent-ils la recherche d'une forme spectaculaire idéale? Sports et théâtre ont par ailleurs en commun la recherche d'une même qualité cognitive: l'impression du vivant. Plus près de nous, des entreprises comme celle du Théâtre Équestre Zingaro ne font-elles pas du sport et du théâtre les deux parties d'une même nature? La mise en piste de pratiquants de kalaripayatt (gymnastique et art martial du Kerala) orchestrés par Pierre Boulez dans le spectacle Triptyk (2000) par Bartabas est un cas exemplaire de cette hybridité. Pour cette performance en trois parties sur Le Sacre du Printemps de Stravinsky, le Dialogue de l'ombre double de Boulez puis La Symphonie de Psaumes de Stravinsky, créée le 17 mars 2000 à Amsterdam en Hollande, Bartabas et la troupe du Théâtre Zingaro accueillent sept pratiquants de kalaripayatt, âgés entre 20 et 30 ans, venus spécialement de l'État du Kerala, au sud de l'Inde (Salino, 1999). Formé dès l'enfance par cet art d'éduquer et d'exercer le corps, que les journalistes considèrent comme l'origine des arts martiaux (Denaud, 1996), le corps des sept gymnastes, sportifs et virtuoses, est fortement, mais sèchement musclé, longiligne, souple et totalement délié, de telle sorte qu'à l'œil, son arrogante et fascinante beauté guerrière 
peut rivaliser avec la puissance des chevaux. La danse martiale des gymnastes constitue la première variation du spectacle et évoque un rite primitif: "Toute la force de cette première partie repose sur le mouvement collectif ; le rythme d'ensemble compte plus que l'exploit individuel" (Solis, 2000, s.p.). Rite, sport et théâtre sont foncièrement distincts (Hamayon, 2012), mais dès qu'un groupe de corps nus en mouvements harmonieusement développés agit avec rythme, vitesse et élégance sur scène ressurgit alors l'idée évolutionniste d'une origine rituelle du spectacle vivant (Lorelle, 2003). Image d'autant plus vive lorsque le spectacle est un habile assemblage d'exotisme, d'animalité, de sport et de théâtre.

\section{Le Sport comme Préparation aux Métiers du Théâtre}

De nombreux travaux en études théâtrales pointent l'appropriation des sports par le milieu des arts vivants de la scène, au début du $\mathrm{XX}^{\mathrm{e}}$ siècle, en Russie, à travers les travaux de Stanislavski sur les actions physiques (Autant-Mathieu, 2007) et ceux de Meyerhold sur son système biomécanique où entrent en jeu toute une variété d'exercices éducatifs, mais aussi d'exercices d'application: sauter, porter, lancer, se défendre, etc. (Picon-Vallin, 2009). Toutefois, assez peu de recherches complémentaires, interdisciplinaires ou pluridisciplinaires, entre études théâtrales et études sportives sont menées pour analyser ces expériences dans leur contexte. Au point de vue des études sportives, nous pouvons nous demander comment les expériences menées par Stanislavski et par Meyerhold s'inscrivent dans les plans d'organisation de l'éducation physique de la masse en Russie? Et, inversement, l'analyse de ces plans est-elle susceptible de nous renseigner sur les expériences de ces réformateurs? À titre comparatif, en France, les transformations opérées par Charles Dullin (Lorelle, 2007) ou avant par Jacques Copeau dans la formation du comédien (Aliverti, 2009) ne se comprennent qu'en tenant compte du rôle majeur joué par le lieutenant de vaisseau Georges Hébert (Philippe-Meden, 2012) dans l'évolution des idées sur le sport, la gymnastique et l'éducation physique. Le propos d'Hébert préfigure aussi "[...] la conception de l'exercice dans le training de l'acteur des années soixante-dix" (Pradier, 2000, p. 74). Eugenio Barba analyse l'intérêt de la Méthode Naturelle d'Hébert dans le training des réformateurs du théâtre: 


\section{( Estudos da Presenca

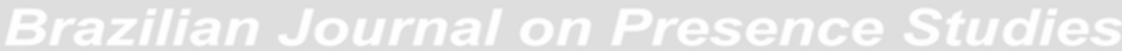

Certaines méthodes gymniques et certains sports ont un rapport direct avec la pratique théâtrale car ils contribuent à développer une capacité de réactions immédiates qui agissent sur le sens kinesthésique des spectateurs. De plus, ils enseignent à l'acteur comment maîtriser les fondements physiques de son métier, dans sa dimension individuelle (l'utilisation de l'énergie), dialogique (la relation avec ses partenaires) et publique (la relation avec les spectateurs). [...] C'est pour ces raisons que Jacques Copeau a choisi la 'gymnastique naturelle' de Georges Hébert comme base du travail de l'acteur de préférence à l'eurythmique de JaquesDalcroze et à la danse (Barba, 2008, p. 115).

Lieutenant de vaisseau de la marine française, réformateur de la gymnastique au Bataillon des Fusiliers Marins en 1904 et directeur du Collège d'Athlètes de Reims (1912-1914), Georges Hébert (18751957) est le promoteur de la Méthode Naturelle d'Éducation physique, virile et morale, introduite chez Jacques Copeau, à l'École du Vieux Colombier, en 1920. La Méthode Naturelle est conçue d'après les dix groupes d'exercices pour lesquels le corps humain est spécialement organisé: marcher, courir, sauter, grimper, porter, lancer, se défendre et nager, auxquels s'ajoutent la quadrupédie et l'équilibrisme.

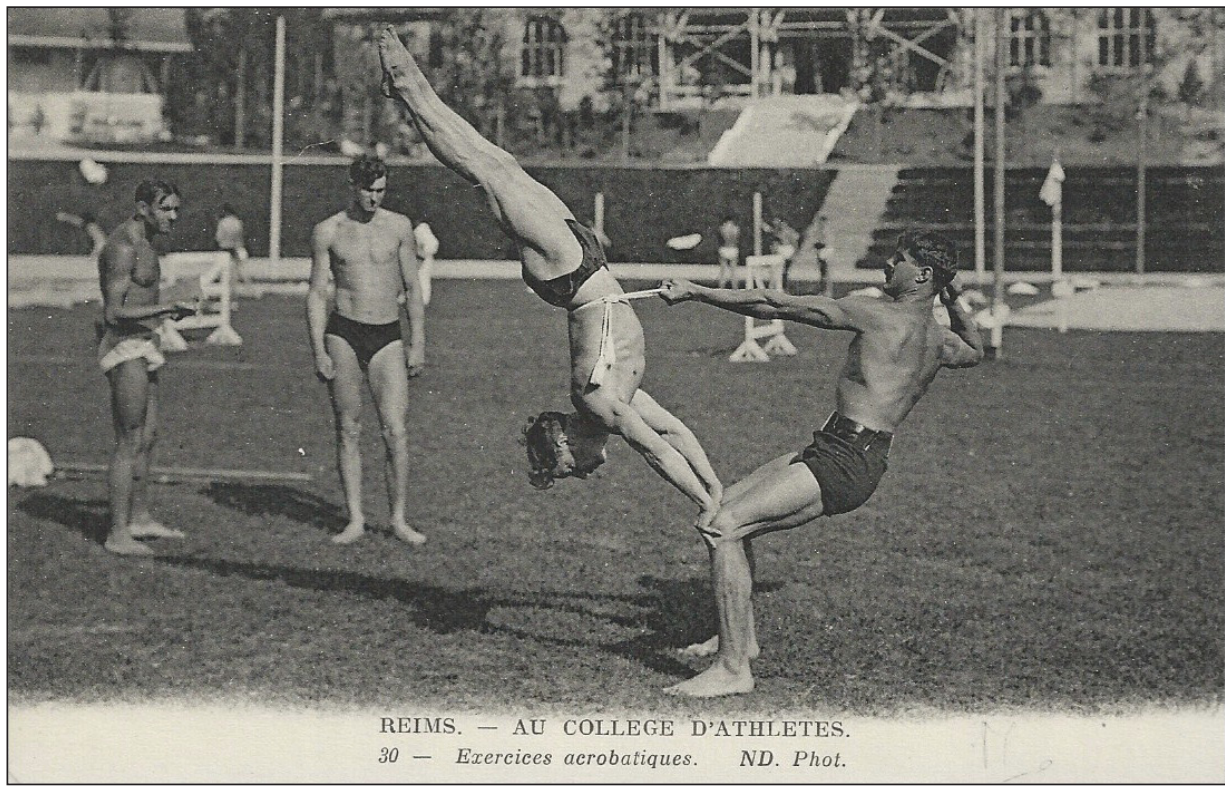

Image 1 - Carte postale représentant un exercice acrobatique au Collège d'Athlètes de Reims. Collection personnelle de l'auteur P. Philippe-Meden.

Grand chef des moniteurs du Collège d'Athlètes de Reims, le gymnaste Louis Moine (1885-1932) transmet la Méthode Naturelle à l'École du Vieux Colombier avec la mission spéciale de former Jean Dorcy qui à terme doit lui succéder. Dorcy, sous la direction de Moi- 


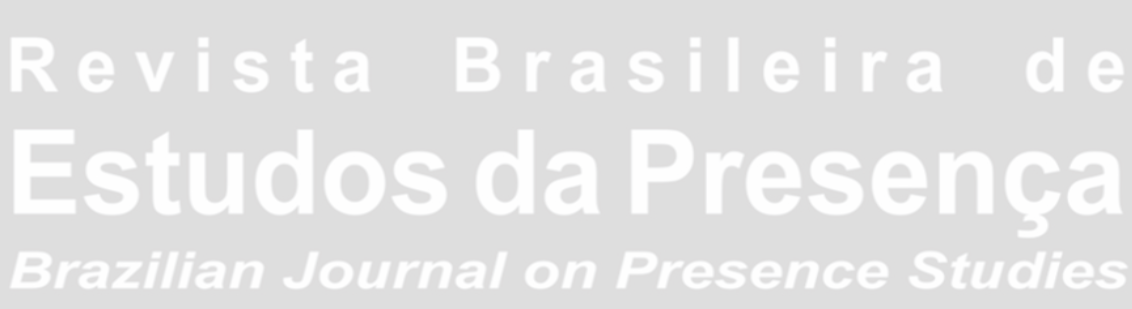

ne, transmet l'hébertisme chez Charles Dullin, à l'Atelier, entre 1922 et 1924. Méthode Naturelle et hébertisme ne sont pas synonymes. L'hébertisme est un modèle holistique de transmission des techniques du corps élaboré par les monitrices hébertistes formées par Hébert au Collège d'Athlètes de Reims et qui s'institutionnalisent en communauté d'athlétesses, à La Palestra - Collège gymnique féminin et enfantin - de Deauville-sur-Mer, en 1919.

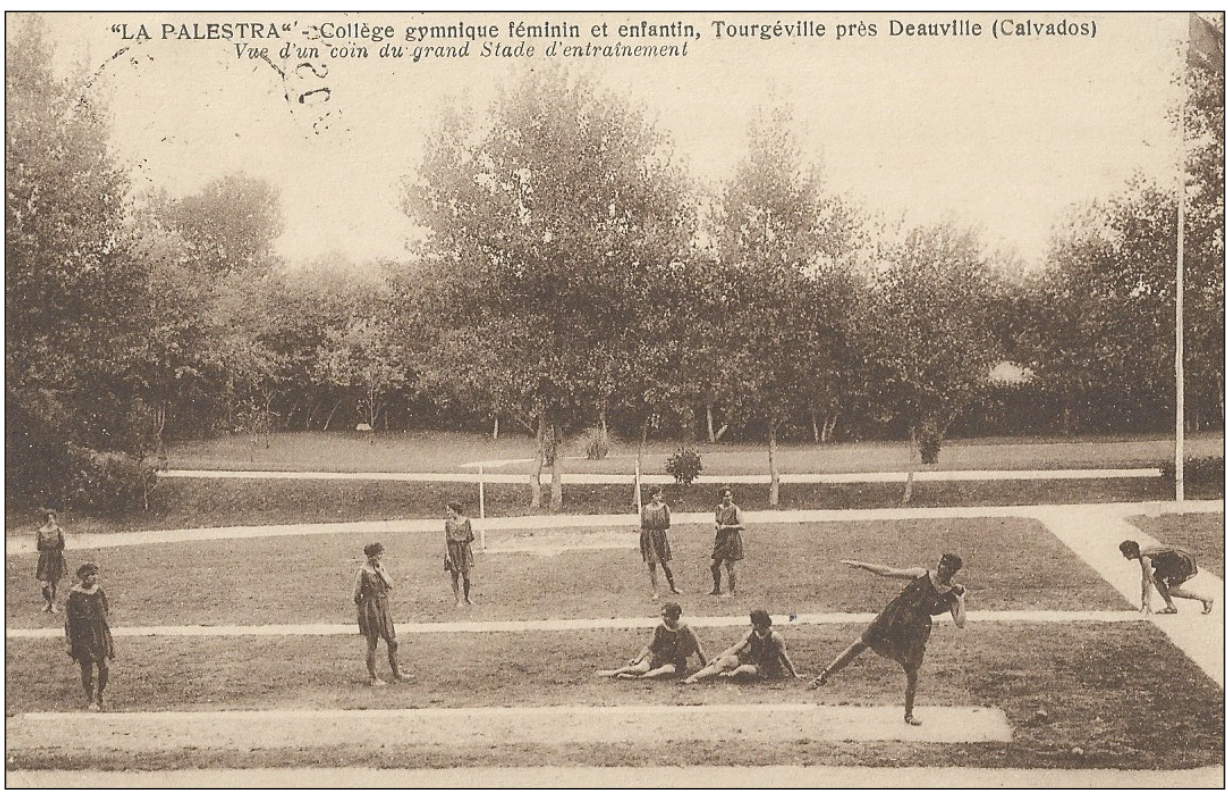

Image 2 - Carte postale représentant un coin du stade de La Palestra.

À remarquer la tenue des athlétesses qui peut évoquer l'imaginaire autour des danseuses d'Isadora Duncan. Collection personnelle de l'auteur P. Philippe-Meden.

Le modèle pédagogique hébertiste se compose de six éléments: 1) un entraînement physique complet (Méthode Naturelle d'éducation physique, sports, cirque, danse et musique); 2) un apprentissage de tous les métiers manuels courants (jardinage, terrassement, maintenance...); 3) une culture mentale et morale (gymnastique psychique de Jean des Vignes Rouge, relaxation, méditation, spiritualisation de l'exercice); 4) une culture intellectuelle (cours, conférences et discussions sur les pratiques corporelles en relation avec le féminisme de Suzanne Grinberg, l'histoire, la géographie, la politique nationale et internationale); 5) une culture esthétique (cours, conférences et discussions sur la beauté plastique, la philosophie mystique et atlantéenne de Paul le Cour, les arts et les arts du spectacle vivant); 6) une initiation naturiste suivant les principes de Paul Carton (dévêtissement, traitement par l'exercice physique, massage, végétarisme, héliothérapie, aérothérapie, hydrothérapie). 
L'hébertisme connaît un rayonnement national et international considérable jusqu'en 1955. Mais, en France, dans les années 19601970, la doctrine du sport éducatif fait peu à peu rejeter l'hébertisme et la Méthode Naturelle aux oubliettes de l'histoire. Toutefois, au début du $\mathrm{XXI}^{e}$ siècle, en réponse à la prédominance des nouvelles technologies qui pouvaient laisser croire à une moindre importance de la composante humaine dans certains métiers et aux conséquences du sédentarisme (cancers du sein et du côlon, maladies cardiovasculaires, diabète), ainsi qu'à l'intérêt renouvelé pour les activités physique de plein air (APPN), l'hébertisme fait un retour en force dans le monde civil à travers les cultures sportives alternatives: Parkour, Freerunning, Challenge Urbain, Crossfit, MovNat et Paléo-fitness et dans le monde militaire auprès des experts en Entraînement et Éducation Physique Militaire et Sportive (E2PMS).

Conjointement à l'influence de l'hébertisme chez les réformateurs du théâtre français, l'influence des sports, notamment de la boxe, chez ces mêmes réformateurs (Ruffini, 1994) est symptomatique de changements sociaux inscrits dans une histoire du corps. Les mimes formés à l'École du Vieux Colombier puis à l'Atelier en témoignent. Étienne Decroux écrit: "[...] autour de 1908 [...] l'obésité n'est plus une référence, l'habileté prime la force, l'homme mince fait son entrée [...] C'est Georges Carpentier [...] Il est bien loin de se douter qu'il fut l'image motrice de notre mime du corps (section tragédie)" (Decroux, 1994, p. 32-33). Jean-Louis s'en fait l'écho: "[...] interpréter Hamlet, par exemple, c'est perdre un kilo et demi dans la soirée, Scapin fait monter la tension de 6 à 7 points. La pulsation des acteurs, comme celle des sportifs, est lente. Être acteur, c'est pratiquer un sport qui exige un équilibre musculaire, respiratoire et nerveux" (Barrault, 1959, p. 35). Depuis l'introduction de la gymnastique dans les cours de la formation du comédien et surtout depuis les travaux de Jerzy Grotowski, notamment avec le yoga (Temkine, 1968), de multiples méthodes reposent sur l'interprétation de cet esprit sportif: entraînement psychophysique, théâtre physique etc. L'argument du théâtre comme outil de médiation sociale et de bien-être devient essentiellement efficace au point de vue commercial. De nombreux cours de théâtre corporel s'ouvrent, mais quelle valeur éducative donner aujourd'hui à la surenchère sportive dans le milieu du théâtre? Par ailleurs, dans d'autres cultures où la pri- 
mauté du texte et de la parole est moins opérante, la formation de l'acteur ne suscite aucun questionnement sur l'influence des sports, en raison d'une apparition tardive de cette notion, comme en Orient par exemple. Il nous faut rappeler que celle-ci, dans le sens où nous l'entendons dans la zone Euro-américaine, n'est apparue en Chine qu'à la fin du XIX ${ }^{\mathrm{e}}$ siècle (Boucher, 2008). En Chine, l'entretien du corps a été une préoccupation importante, principalement dans les milieux taö̈stes, qui ont fait leur apanage de la quête de longévité. Ainsi va se développer: pratique physique (arts martiaux, taijiquan, qigong), diététique, médecine, etc. Selon Catherine Despeux, la pratique corporelle "[...] privilégiait en effet la lenteur, la modération, la souplesse et la simplicité du mouvement dans le taijiquan et le qigong [...], méthodes les plus en vogue encore à l'heure actuelle" (Despeux, 2004 , p. 46). Nous sommes loin de la pratique sportive à la recherche de la performance, de la dépense d'énergie d'un effort soutenu. La conception holistique du monde se retrouve aussi dans le jingju: les catégorisations artistiques tout comme le paradigme de la séparation de l'esprit et du corps sont inopérantes. Le performeur doit, à la fois savoir chanter, danser, se battre et exécuter des actions mimées. Ainsi, son entraînement, commencé dès l'âge de 8 ans d'une durée de dix ans, est foncièrement gymnique afin d'assouplir son corps et lui faire maîtriser l'acrobatie et la gestuelle codifiée. L'idée de lutte, d'effort soutenu et de danger à vaincre pour surprendre le public est omniprésente dans sa formation. D'un point de vue extérieur, nous serions tentés de parler d'entrainement sportif, bien qu'il ne soit pas pensé comme tel.

\section{L'Identité Naturiste entre Sport et Théâtre}

Le mythe du "retour à la nature" (Baubérot, 2004; Villaret, 2005) joue-t-il un rôle dans l'hybridation de l'activité théâtrale et des sports? La popularisation des sociétés de gymnastique et des activités artistiques connexes aux sports dans l'Allemagne de la Belle Époque semble déjà l'indiquer (Colomer, 1997). La quête de l'acteur sain chez les réformateurs du théâtre français par l'introduction de l'hébertisme dans les cours de la formation du comédien pourrait aussi évoquer l'idéologie du surhomme, du surhomme nietzschéen ou de "[...] l'homme nouveau dans l'Europe fasciste" (Matard-Bonucci; Milza, 2004): 
Les quelques dix milles ans de civilisation diverses ont bien aussi leur empreinte, leur histoire physiologique, leur philosophie, leur concourante et leurs réactions. Dans le chaos de cet infini, il a bien fallût qu'il [Hébert] fit un choix. Mais s'il taille dans l'infini, au moins ne prétend-il point ni l'enfermer tout entier dans sa formule, ni le réduire à quelques comprimés de mouvements. On a tant vaticiné sur le détail optimum que c'est un grand soulagement d'entendre enfin un rénovateur replacer la question en face du gigantesque projet global: sans rien perdre des acquis, ramasser ce que nous perdons de nos hérédités et lancer l'homme renouvelé à l'assaut du surhomme physique; et comme chemin, se vérifier sans cesse vers le but, lequel consiste dans l'évaluation des résultats de production. Voilà bien un plan de bataille de vie. Celui qui l'a créé et en a réalisé une exécution est en tête de son idée sans qu'on puisse l'inféoder à aucune autre école (Lhermitte, 1914, p. 45).

Les APPN apparaissent tandis que s'ouvrent les nombreux théâtres du peuple, théâtres de plein air et théâtres de la nature. Sociétaire de la Comédie Française, Béatrix Dussane ne conçoit pas de meilleure éducation pour l'enfant et de meilleur entraînement pour le comédien que de la marche en montagne. Dans un cours sur Les Femmes de Molière prononcé à la Société des Conférences, à Paris, les 13, 20 et 27 mars 1931, elle se réfère à l'un des spectacles de la nature que "[...] seuls peuvent décrire et comprendre ceux qui se sont donné la peine physique de le rechercher":

Lorsqu'on marche en montagne avant l'aurore, on voit de ces illuminations. Au-dessus des sombres pentes boisées et de l'encre des roches, le sommet neigeux que l'aube glace encore apparaît gris, décoloré, hostile. Tout à coup, par l'échancrure d'un col lointain, le premier rayon du soleil a passé. Dans un éclair, les livides névés s'enflamment, c'est un ruissellement de nacre et de rose, c'est la promesse étincelante de toutes les joies, de toutes les fécondités du jour. C'est ce feu d'aurore que je voudrais voir sur la neige du visage d'Agnès (Dussane, 1933, p. 37).

La marche en montagne fait du corps un instrument souple, robuste et obéissant, et amène à une plénitude de connaissance et de pensée qui conduit à “[...] cette liberté de l'âme et de l'esprit, la seule que permet un corps sans entraves, une respiration parfaite, une absence de souci digestif ou articulaire" (Chardon, 1934, p. 146). La montagne est donc pour Dussane “[...] l'éducatrice morale, intellectuelle et physique du vrai comédien et de la bonne comédienne" (Dussane, 1934, p. 253). La montagne offre encore des exercices 
de grimpers sur les rocs, des courses le long des pentes, des sauts sur accidents de terrain et le porter du sac sur le dos, autrement dit pour Dussane: l'entraînement hébertiste mis à la mode par les scouts (Dussane, 1934, p. 254).

Au point de vue technologie des exercices, les leçons d'hébertisme données aux louveteaux sont des séquences d'actions dramatisées qui se rapprochent de ce que préconise Léon Chancerel. Par exemple, dans Les Livres de la Jungle, de Kipling, un épisode rapporte que Mowgli est recueilli par Mère louve puis accepté par le clan. L'objectif est de suivre Mowgli, de faire comme lui. Mowgli s'éveille au milieu de ses frères loups. Sa faim retentit. Tous partent en chasse:

Marche: Lentement, pour ne pas brouiller les pistes, on marche en relevant des traces, sur le sol, puis dans les branches: feuilles arrachées, toisons accrochées... Voici enfin une piste d'Antilopes. Course: Les proies sont en vue, mais l'une d'elles a pris l'alarme et toutes s'enfuient. Nous les suivons en rampant ou à quatre pattes dans les buissons, en course de vitesse en terrain libre. Saut: Les Antilopes ont sauté une rivière, puis un fossé, des buissons, des lianes; la Meute saute après elle, par-dessus des cordes tendues par le Chef. Lutte: Les herbivores se défendent, avant de succomber sous les crocs du clan. Lever-Porter: Après s'être rassasié, on lève les restes, on les charge, pour les porter dans une cache, où on les conservera pour les jours de famine. Grimper: Mowgli, altéré par la chair fraîche, grimpe dans les arbres, pour cueillir des fruits: noix de coco, dattes ou bananes. Lancer: Revenu à terre, il s'amuse à jeter de plus en plus loin les débris de son dessert (Bracquemart, 1930, p. 277).

Par ce type de leçon-séquences, les louveteaux sont amenés à vivre réellement les différentes actions de la Méthode Naturelle d'une manière divertissante qui éveille l'imagination.

Le scoutisme est pour Léon Chancerel une terre d'accueil où il concrétise sa vision du théâtre, avec les Comédiens Routiers, d'après les principes de Jacques Copeau dont il est l'élève à l'École du Vieux Colombier (Romain, 2005). Dans son livre sur Le Théâtre et la jeunesse, Chancerel écrit que la Méthode Naturelle est celle qui se rapproche le plus de celle qu'il préconise: "Gymnastique d'abord. Mais pas n'importe quelle gymnastique : une gymnastique orientée vers l'expression dramatique. [...] Chaque éducateur inventera facilement des fictions et des personnages destinés à dramatiser les divers éléments de la leçon-type d'Hébertisme" (1941, p. 35-36). Le lien 
entre Chancerel et l'hébertisme n'est pas sans malentendus (Page, 2009), notamment lorsqu'il rapproche Hébert d'une idéologie nationale-socialiste. La posture d'Hébert face à l'éducation physique en Allemagne se distingue nettement de la posture de Chancerel pour le théâtre. Le milieu hébertiste surveille l'évolution de l'éducation physique en Allemagne depuis la signature du Traité de Versailles (1919), mais l'opinion d'Hébert se radicalise après l'arrivée d'Hitler au pouvoir: "[...] sous la dictature hitlérienne. On décide une violente réaction contre les sports de compétitions et, plus que jamais l'entraînement de la MASSE par un retour aux doctrines de F.-L. Jahn" (L'Éducation Physique, 1934, p. 18)3. Le but d'Hébert n'a jamais été de "[...] faire des êtres forts" (L'Éducation Physique, 1934, p. 179) à la manière des culturistes ou des fervents de la culture du corps, car pour lui le corps est le temple de l'âme et du cerveau. Et plaçant l'altruisme à la base de sa doctrine, son objectif est de contribuer à éduquer, développer et entretenir en chacun les moyens d'action et d'adaptation utiles pour se porter secours ou porter secours à autrui dans toutes les circonstances. La Méthode Naturelle s'adresse à tous. Contrairement aux idées reçues, Hébert ne s'oppose pas au corps médical, mais au scientisme. Quelles sont les compétences d'un médecin dans le domaine de l'éducation physique? D’après Hébert le médecin ne peut se substituer au gymnaste: celui qui enseigne les exercices du corps. Sa devise qu'il inscrit sur la couverture de la revue L'Éducation Physique est explicite: "L'éducation physique doit être une œuvre scolaire. Aux Maîtres de la réaliser"! On peut encore se demander d'où viennent ces étonnantes considérations: Hébert "homophobe" (L'Éducation Physique, 1934, p. 181) et sa méthode "eugéniste" et "particulièrement dure" (L'Éducation Physique, 1934, p. 182)! Enfin, sous Vichy, la Méthode Naturelle est bien la référence officielle, mais Hébert s'est séparé du milieu hébertiste au début de la guerre de 1939-1945 et son nom ne peut donc être associé aussi simplement à la politique sportive du gouvernement de Vichy. Aussi faudrait-il se questionner sur l'obligation que peut avoir Chancerel de citer Hébert ou plutôt l'hébertisme dans son ouvrage sur le théâtre et la jeunesse paru en 1941. À cette époque, l'État fait pression pour l'imposer.

Sports, APPN, danses, jeux et chants, familiarisent les scouts aux arts vivants de la scène. C'est chez eux que l'Éducation Par le 
Jeu Dramatique (EPJD), association fondée en 1946 par l'hébertiste Jean-Marie Conty, recrute après la guerre de 1939-1945. Conty y souligne la valeur d'une expression scénique basée sur une méthode globale d'éducation physique et psychique favorisant des mouvements spontanés ou improvisés plutôt que la restitution de gestes appris. L'éducation physique à l'EPJD ne vise pas directement les performances athlétiques ou sportives, mais le développement de qualités physiques telles que souplesse, coordination, amplitude et rythme du geste, décision et rapidité des réflexes. En hébertiste convaincu, Conty se rattache à ce principe qu'il serait préférable que cette éducation physique fasse partie de l'éducation générale et se répartissent de l'enfance à l'âge adulte: "[...] alors il s'agirait d'une évolution naturelle vers la perfection de l'être affectif et physique, et non plus d'une rééducation d'adolescents ou d'adultes déjà à demi paralysés par une éducation faussée" (Conty, 1947, n/p). Ami de Conty et de Barrault, le pédagogue Jacques Lecoq fonde son École Internationale de Théâtre en 1956, à Paris, où il développe une nouvelle pédagogie pour la formation du comédien qui repose sur la transposition de sa pratique de l'hébertisme dans un mime d'action:

[...] je pratiquais la méthode naturelle de Georges Hébert: tirer, pousser, grimper, marcher, courir, sauter, lever, porter, attaquer, se défendre, nager. Ces actions tracent dans le corps sensible des circuits physiques dans lesquels s'inscrivent des émotions. Des sentiments, des états et des passions s'expriment à travers des gestes, des attitudes et des mouvements analogiques à ceux des actions physiques. Il est important de savoir comment le corps 'tire', comment il 'pousse', afin de pouvoir, le cas échéant, exprimer toutes les manières particulières de 'tirer' ou de 'pousser' d'un personnage. Analyser une action physique, c'est appréhender une connaissance, base indispensable pour le jeu (Lecoq, 1997, p. 82).

Par ailleurs, d'autres éducateurs physiques, à l'instar d'Yves Marc fondateur du Théâtre du Mouvement, effectuent le retour aux sports par l'organisation de stages en pleine nature et spécialement de marche en montagne. Le naturisme se conçoit donc comme un facteur apte à favoriser les transferts de connaissance entre milieu du théâtre et milieux sportifs. Le passé oublié d'éducateur physique chez de nombreux réformateurs des arts vivants de la scène, danse et théâtre, révèle aussi l'importance d'études biographiques pour mieux saisir leurs créations. Le butô de Kazuo Ono peut-il se comprendre 
sans l'analyse de l'influence de la gymnastique allemande dans son passé d'éducateur physique, dans un collège pour jeunes filles? Comment l'esprit sportif se transpose-t-il du gymnase au théâtre? Enfin, l'identité de pratique entre sports et théâtre n'est pas sans poser un certain nombre de malentendus pour l'étude d'objets hybrides comme le match d'improvisation théâtrale (Ertel, 1985), le kalaripayatt (Philippe-Meden, 2005) ou la capoeira (Garrabe, 2013). Le match d'impro ressort-il du sport ou du théâtre? Pourquoi la capoeira est-telle plus étudiée en anthropologie et en études théâtrales plutôt qu'en études sportives? De même, le kalaripayatt est-il de la gymnastique, un art martial ou de la danse (Silvestri, 2013)? Le kalaripayatt, principalement objet d'analyse en études théâtrales, est pourtant un facteur essentiel du tourisme sportif et du bien-être au Kerala. Autant d'objets qui, pour être abordés, nécessitent la complémentarité des compétences en études sportives et en études théâtrales.

\section{Théâtre Sportif ou Educatif?}

Une littérature sportive (Rozet, 1914) naît au début du XX ${ }^{\mathrm{e}}$ siècle avec les Essais de psychologie sportive (Coubertin, 1913) puis Les Chants du Stade (Souchon, 1923), Le Paradis à l'ombre des épées (Montherlant, 1923), les récits sportifs de Bertolt Brecht (1922-1956)... À côté des journaux, magazines et revues qui inondent les kiosques de comptesrendus de matches et de championnats, cette littérature prend pour source d'inspiration la vie musculaire, les sensations et les attitudes du boxeur, du footballeur, de l'escrimeur ou du cavalier, avec le but de les élever à la dignité des belles-lettres. Elle renseigne sur le goût des sportifs, et indique qu'ils ne sont pas des barbares désintéressés de la parole écrite sous formes de contes et de récits. Un théâtre sportif à l'image de cette littérature émerge également à cette période. Par exemple, la pièce Little Palace, c'est couru nous documente sur les dessous du turf, caricature les propriétaires d'écuries de courses, les entraîneurs et les jockeys, mais contient aussi une scène dans laquelle "deux boxeurs nous font assister aux angoisses du swing et du knock out" (Pontié, 1908, n/p). Si les années 1920-1930 sont pour l'esprit sportif le début du dévoiement vers le professionnalisme, l'argent, la spécialisation et l'exhibition, les réformateurs français du théâtre opèrent une moralisation du théâtre contre l'histrionisme et le cabotinage. Le théâtre qu'ils recherchent doit être éducatif et représenter 
une éducation globale pour l'enfance et la jeunesse. Considération qui force à s'interroger sur la distinction entre le sens du sport et celui de l'éducation physique. Les gens de théâtre évoquent indistinctement les mots et expressions: sport, athlétisme, gymnastique, éducation physique et culture physique, mais tous renvoient à des pratiques et des idéologies différentes et clairement définies par le milieu hébertiste. Par exemple, le sport fait référence à:

Sport - Tout genre d'exercice ou d'activité physique ayant pour but la réalisation d'une performance et dont l'exécution repose essentiellement sur l'idée de lutte contre un élément défini : une distance, une durée, un obstacle, une difficulté matérielle, un danger, un animal, un adversaire et par définition soi-même (Hébert, 1921, p. 6-7).

La performance est l'essence du sport, aussi un comédien qui cherche à améliorer les qualités physiques de sa présence, de son geste ou de sa voix fait-il du sport, mais comme l'observe Georges Banu:

Le théâtre suscite rarement la perception 'sportive' du corps. Car ici le corps peut être entraîné sans qu'il soit pour autant perçu comme performant. (Parfois, et cela est désagréable, le corps s'institue abusivement en objet central du spectacle, comme dans une compétition: le théâtre n'est pas un sport) (Banu, 1985, p. 64).

Par contre, observe-t-il encore, à l'Opéra, ajoutons au cirque ou au Jingju, les sensations peuvent êtres plus semblables à celles éprouvées lors d'un matche puisque les spectateurs viennent assister aux prouesses du corps. Et, cependant, la recherche esthétique propre aux arts du spectacle vivant transcende la performance sportive et rejette l'impression d'effort aux jeux du training ou aux jeux du stade:

Grotowski raconte que lors d'un spectacle de l'Opéra de Pékin on acclamait les performances du père, tandis qu'on faisait un accueil plus que réservé au fils. Lui-même narrivant pas à distinguer la qualité de leur art, il interrogea quelqu'un qui lui répondit: 'C'est que le fils transpire, tandis que le père non'. La sueur, dans les théâtres traditionnels, en rendant trop visible l'effort, disqualifie la performance: elle perd sa vocation impersonnelle et le corps de l'acteur l'investit abusivement (Banu, 1986, p. 101).

D'un autre côté, l'éducation physique vise le développement intégral du corps, l'harmonisation des fonctions et des formes, la santé et la beauté:

Éducation physique - Action méthodique, progressive et continue, de l'enfance à l'âge adulte, ayant pout objet 
ISSN 2237-2660

d'assurer le développement physique intégral: d'accroître les résistances organiques; de mettre en valeur les aptitudes dans tous les genres d'exercices naturels et utilitaires indispensables [...]; de développer l'énergie et toutes les autres qualités d'action ou viriles; enfin de subordonner; tout l'acquis, physique et viril, à une idée morale dominante: l'altruisme (Hébert, 1934, p. 12-13).

L'éducation physique s'adresse à un pédagogue auquel la seule pratique des exercices du corps ne suffit pas puisque le développement intégral de l'individu suppose aussi une alimentation raisonnée, le réglage du travail et du repos, la façon de se vêtir... L'éducation physique chez les réformateurs français du théâtre implique un art de vivre que la vie en communauté, école ou compagnie, favorisera. Le sport n'est pas un moyen éducatif, car la spécialisation qu'il demande pour le record engendre un défaut d'harmonie dont les conséquences peuvent être fâcheuses: déchirures musculaires, déformations vertébrales, fractures osseuses. L'éducation physique s'arrête où commence le spectacle vivant. Le sport et le théâtre peuvent couronner une éducation bien pensée, sans s'y substituer. 


\section{Notes}

${ }^{1}$ Le spectacle de masse chorégraphié par Laban sera annulé, car jugé "trop intellectuel" (Guilbert, 2000, p. 231).

${ }^{2}$ Pendant l'occupation de la France par l'Allemagne, le commissariat Borotra (1940-1942) cherche à définir "[...] une méthode nationale susceptible de refonder le programme de l'École et de l'Université [...]. C'est dans cette perspective, ce projet, que se vit intégrée au programme nouveau la Méthode Naturelle de Georges Hébert" (Gay-Lescot, 1995, p. 27). Larrêté du 16 août 1941 fait que les leçons d'Éducation physique doivent êtres données suivant les principes de la Méthode Naturelle. Mais, dans les faits, Hébert n’est pas associé, ni même consulté: "L'idée d'une rencontre ou d'une collaboration avec Georges Hébert, si elle avait été évoquée, n’avait pas dépassé le stade d'hypothèse. [...] et il n'y eut [...] aucune rencontre entre l'auteur de la Méthode Naturelle et les autorités de Vichy" (Gay-Lescot, 1995, p. 25).

${ }^{3}$ Friedrich-Ludwig Jahn (1778-1852), le Turnvater, est l'inventeur d'une méthode d'éducation physique destinée à combattre les français.

\section{Références}

ALIVERTI, Maria-Inès. Jacques Copeau, Artigiani di una Tradizione Vivente. L'attore e la pedagogia teatrale. Firenze: Volo, 2009.

ARTAUD, Antonin. Un athlétisme affectif. In: ARTAUD, Antonin. Le Théâtre et son Double. Paris: Gallimard, 1964 [1935]. P. 199-211.

AUTANT-MATHIEU, Marie-Christine. La Ligne des Actions Physiques. Répétitions et exercices de Stanislavski. Montpellier: L'Entretemps, 2007.

BANU, Georges. L'Acteur qui ne revient pas. Journées de théâtre au Japon. Paris: Gallimard, 1993 [1986].

BANU, Georges. Le spectateur: le stade et la scène. Théâtre/Public, Gennevilliers, Théâtre de Gennevilliers, n. 63, p. 62-64, 1985.

BARRAULT, Jean-Louis. Sport et représentation dramatique. L’Éducation Physique, Paris, Fédération Française d'Éducation Physique, p. 35-39, 1959.

BAUBÉROT, Arnaud. Histoire du Naturisme. Le mythe du retour à la nature. Rennes: Presses Universitaires de Rennes, 2004.

BONAL, Gérard. Les Renaud-Barrault. Paris: Seuil, 2000.

BOUCHER, Aurélien. Le sport en Chine au début du XX $\mathrm{XX}^{\mathrm{e}}$ siècle. Perspectives Chinoises, Hong Kong, Centre d'Étude Français sur la Chine Contemporaine (CEFC), n. 2008/1, 2008. Disponible sur: <http://perspectiveschinoises.revues.org/3753>. Consulté le: 21 fév. 2012.

BRACQUEMART, Thérèse. Scoutisme. Comment on peut faire de l'Éducation physique par la Méthode Naturelle dans une Meute Scout de France. In: HÉBERT Georges (Org.). L'Éducation Physique, Paris, n. 16, p. 277-278, 15 oct. 1930. 
BRECHT, Bertolt. L’Uppercut et Autres Récits Sportifs. Paris: L’Arche, 2006 [1922-56]. BROMBERGER, Christian. Football, la Bagatelle la plus Sérieuse du Monde. Paris: Bayard, 1998.

CHANCEREL, Léon. Le Théâtre et la Jeunesse. Paris: Bourrelier \& C ${ }^{\text {ie }}$, 1941 [1939].

CHARDON, Pierre. Notes littéraires. In: HÉBERT, Georges (Org.). L'Éducation Physique, Paris, n. 30, p. 145-147, 15 avr. 1934.

CHENG, Anne. Histoire de la Pensée Chinoise. Londrai: Seuil, 2002 [1997].

COLOMER, Henry. Monte Verita, l'Utopie d'un Nouvel Âge. Paris: AMIP/ARTE, 1997.

CONTY, Jean-Marie. Le jeu dramatique II - Entraînement de l'acteur athlète effectif. La Maison des Jeunes, Neuilly-sur-Seine, La Fédération des Maisons des Jeunes, n. 20, n/p, avr./mai. 1947.

COUBERTIN, Pierre de. Renaissance chorégraphique. In: VIENNE, Théodore (Org.). L'Éducation Physique, Paris, n. 6, p. 158-159, 1913.

DANIEL, Laurent (Org.). L'Art et le Sport, Actes du XII ${ }^{\mathrm{e}}$ Colloque International du Comité Européen pour L'Histoire des Sports - Lorient 2007. 2 tomes. Biarritz: Musée National du Sport/Atlantica, 2009.

DARROBERS, Roger. Opéra de Pékin - théâtre et société à la fin de l'Empire Sino Manchou. Paris: Bleu de Chine, 1998.

DECROUX, Étienne. Paroles sur le Mime. Paris: Librairie Théâtrale, 1994 [1963].

DENAUD, Patrick. Kalaripayat. L'origine des arts martiaux. Paris: Budostore, 1996.

DESPEUX, Catherine. La gymnastique Daoyin 導引 dans la Chine ancienne. Études Chinoises, Paris, AFEC, v. 23, p. 45-86, 2004.

DURAND-DASTES, Vincent. Le fantôme d'une belle. Le théâtre chanté chinois (xiqu戏 曲) au tournant du millénaire. In: FEUILLEBOIS-PIERUNEK, Ėve (Org.). Théâtre d'Asie et d'Orient - traditions, rencontres, métissages. Bruxelles: Scientifiques Nationales/P.I.E. Peter Lang, 2012. P. 91-120.

DUSSANE, Béatrix. Le Comédien sans Paradoxe. Paris: Plon, 1933.

DUSSANE, Béatrix. À l'École de la Montagne. In: HÉBERT, Georges (Org.). L'Éducation Physique, Paris, n. 32, p. 253-255, 15 oct. 1934.

ERTEL, Évelyne. Le théâtre pris au piège du sport. Ligue Nationale d'Improvisation. Théâtre/Public, Gennevilliers, Théâtre de Gennevilliers, n. 63, p. 74-78, 1985.

FAIVRE, Abel. L'idée de synthèse dans la Méthode Naturelle. In: HÉBERT, Georges (Org.). L'Éducation Physique, Paris, n. 36, p. 14-15, 15 déc. 1925.

GARRABÉ, Laure (Org.). Capoeiras - objets sujets de la contemporanéité. CulturesKairos - Revue d'anthropologie des pratiques corporelles et des arts vivants, Saint-Denis, Maison des Sciences de l'Homme Paris Nord, n. 1, n/p, déc. 2012. Disponible sur: <http:// revues.mshparisnord.org/cultureskairos/index.php>. Consulté le: 11 jan. 2013. 
GAY-LESCOT, Jean-Louis. Le Gouvernement de Vichy et la Méthode Naturelle. In: HÉBERT, Georges. Spirales, Lyon, Centre de Recherche et d'Innovation sur le Sport UFRAPS/Université Lyon I, n. 9, p. 27-30, 1995.

GERNET, Jacques. Le Monde Chinois. Paris: Armand Colin/VUEF, 2003.

GUILBERT, Laure. Danser avec le III ${ }^{\mathbf{e}}$ Reich. Les danseurs modernes sous le nazisme. Paris: Complexe, 2000.

HÉBERT, Georges. Le Sport contre l'Éducation Physique. Paris: Revue E.P.S., 1993 [1925].

L'ÉDUCATION PHYSIQUE. L'Éducation Physique en Allemagne. In: HÉBERT, Georges (Org.). L'Éducation Physique, Paris, n. 29, p. 18-20, 15 jan. 1934.

LECOQ, Jacques. Le Corps poétique, un enseignement de la création théâtrale. En collaboration avec Jean-Gabriel Carasso et Jean-Claude Lallias. Paris: ACTES SUD, 1997.

LENCLUD, Gérard. La tradition n'est plus ce qu'elle était... Sur la notion de "tradition" et de "société" en ethnologie. Terrain, Paris, n. 9, p. 110-123, 1987. Disponible sur: <http:// terrain.revues.org/3195>. Consulté le: 11 jan. 2013.

LHERMITTE. L'Cuvre d'Hébert. In: VIENNE, Théodore (Org.). L'Éducation Physique, Paris, n. 6, p. 43-45, 31 jan. 1914.

LORELlE, Yves. Le Corps, les Rites et la Scène des Origines au XX ${ }^{\text {ème }}$ Siècle. Paris: Les Éditions de l'Amandier/Théâtre, 2003.

LORELLE, Yves. Dullin-Barrault. L'éducation dramatique en mouvement. Paris: Éditions de l'Amandier, 2007.

MATARD-BONUCCI, Marie-Anne; MILZA, Pierre (Org.). L'Homme Nouveau dans l'Europe Fasciste (1922-1945). Entre dictature et totalitarisme. Paris: Fayard, 2004.

MONTHERLANT, Henri de. Le Paradis à l'Ombre des Épées. Paris: Librairie Grasset, 1923.

MÜLLER, Carol (Org.). Le Training de l'Acteur. Paris: ACTES SUD/CNSAD, 2000.

PAGE, Christine. Pratiques Théâtrales dans l'Éducation en France au $\mathbf{X X}^{\mathrm{e}}$ Siècle. Aliénation ou Émancipation?. Arras: Artois Presses Université, 2009.

PHILIPPE-MEDEN, Pierre. Aspects de la Transmission du Kalaripayattu, à travers l'Enseignement de Cécile Gordon et de Philippe Collinet, en France, de 2002 à 2004. 2005. Mémoire (Maîtrise en ÉtudeThéâtral es) - Programme d'Études Supérieures en Ethnoscénologie, Université Paris 8 Vincennes-Saint-Denis, Saint-Denis, 2005.

PHILIPPE-MEDEN, Pierre. Georges Hébert et le Théâtre. Une esthétique de la Nature au fil de l'Éducation Physique, la revue Sportive, Scientifique, Pédagogique, d'Enseignement et de Critique (1902-1940). 2012. Thèse (Doctorat en Études Théâtrales) - Programme d'Études Supérieures en Ethnoscénologie, Université Paris 8 Vincennes-Saint-Denis, SaintDenis, 2012. 
PICON-VALLIN, Béatrice. Meyerhold. Écrits sur le théâtre, Tome 2: 1917-1930. Lausanne: L’Âge d'Homme, 2009 [1975].

PIMPANEAU, Jacques. Promenade au Jardin des Poiriers, l'Opéra Classique. Paris: Musée Kwok On, 1983.

PONTIÉ, Édouard. Au théâtre: Little Palace, c'est couru - Revue sportive en deux actes. In: VIENNE, Théodore (Org.). L'Éducation Physique, Paris, n. 11, p. 308, 15 jui. 1908.

PRADIER, Jean-Marie. Eugenio Barba: l'exercice invisible. In: MÜLLER, Carol (Org.). Le Training de l'Acteur. Paris: ACTES SUD/CNSAD, 2000. P. 57-78.

ROBINET, Isabelle. L'Histoire du Taoïsme, des Origines au XIV Siècle. Saint-AmandMontrond: Cerf CNRS, 2012.

ROMAIN, Maryline. Léon Chancerel. Un réformateur du théâtre français (1886-1965). Lausanne: L'Âge d'Homme, 2005.

ROZET, Georges. Littérature sportive. In: VIENNE, Théodore (Org.). L'Éducation Physique, Paris, n. 4, p. 103-106, 28 fév. 1914.

RUFFINI, Franco, Teatro e Boxe. Latleta del cuore nella scena del Novecento. Bologna: Il Mulino, 1994.

SALINO, Brigitte. Les chevaux de Bartabas s'apprêtent pour un nouveau sacre. Propos de Bartabas recueillis par Brigitte Salino. Le Monde, Paris, 25 déc. 1999. Disponible sur: <http:// www.lemonde.fr>. Consulté le: 3 aoû. 2013.

SILVESTRI, Laura. Corps, Soi, Émotions dans la Pratique Contemporaine des Arts Martiaux Indiens. 2013. Thèse (Doctorat en Anthropologie) - Programme d'Études Supérieures en Anthropologie, Université de Turin, Turin, 2013.

SOLIS, René. Ode pour un cheval défunt. Libération, Paris, 30 mar. 2000. Disponible sur: $<$ http://www.liberation.fr/culture/0101327470-triptyk-le-dernier-spectacle-de-zingaro-marqueune-nouvelle-etape-vers-l-ascese-ode-pour-un-cheval-defunt>. Consulté le: 3 aoû. 2013.

SOUCHON, Paul. Les Chants du Stade. Paris: Monde Nouveau, 1923.

TEMKINE, Raymonde. Grotowski. Lausanne: La Cité, 1968.

VILLARET, Sylvain. Histoire du Naturisme en France, depuis le Siècle des Lumières. Paris: Vuibert, 2005.

ZHANG, Ning. L'Appropriation par la Chine du Théâtre Occidental - un autre sens de l'Occident. Paris: L'Harmattan Littératures, 1998. 
Pierre Philippe-Meden est docteur en études théâtrales de l'Université Paris 8. Chercheur hébergé à la Maison des Sciences de l'Homme Paris Nord, rattaché au laboratoire d'ethnoscénologie et membre de la Société Française d'Ethnoscénologie. Il a enseigné l'histoire du sport et l'anthropologie des pratiques corporelles à la Faculté des Sports et de l'Éducation physique de Liévin, Université d'Artois.

E-mail: pierre.philippe-meden@mshparisnord.fr

Eléonore Martin actuellement en poste d'Attaché temporaire d'enseignement et de recherche à l'Université de La Rochelle où elle enseigne la langue et la civilisation chinoise. Elle est doctorante à l'Université Paris 8. Elle a déjà effectué plusieurs terrains à Taiwan: à l'université nationale Sun Yatsen à Gaoxiong et au sein de la Compagnie Taiwan Yuju et un stage intensif à Beijing Vocational Institute of local Opera and Arts en Chine.

E-mail: eleonore.martin@mshparisnord.fr

Texte original et inédit en français, révisé par Marcio Müller. Une traduction en portugais est publiée dans ce même numéro de la revue.

Reçu le 27 janvier 2013

Accepté le 18 juillet 2013 